\title{
Modular Transport of Postsynaptic Density-95 Clusters and Association with Stable Spine Precursors during Early Development of Cortical Neurons
}

\author{
Oliver Prange, ${ }^{1}$ and Timothy H. Murphy ${ }^{1,2}$ \\ Kinsmen Laboratory, Departments of ${ }^{1}$ Psychiatry and ${ }^{2}$ Physiology, University of British Columbia, Vancouver, British \\ Columbia, Canada V6T 1 Z3
}

The properties of filopodia and spines and their association with the postsynaptic density (PSD) protein PSD-95 were studied during early development of cultured cortical neurons using time-lapse confocal microscopy. Neurons were transfected with recombinant PSD-95 constructs fused to green fluorescent protein (GFP) for, on average, either $8 \mathrm{~d}$ in vitro (DIV) or 14 DIV. We find that, during $1 \mathrm{hr}$ of imaging, filopodia and spines bearing PSD-95/GFP clusters are significantly more stable (i.e., do not turnover) than those lacking clusters. When present within a spine precursor, a PSD-95/GFP cluster appeared to nucleate a relatively stable structure around which filopodium- spine membranes can move. Although processes bearing clusters were generally stable, in 8 DIV neurons, we observed that a subset $(\sim 10 \%)$ of PSD-95/GFP clusters underwent rapid modular translocation between filopodia-spines and dendritic shafts. We conclude that, during early synaptic maturation, prefabricated PSD-95 clusters are trafficked in a developmentally regulated process that is associated with filopodial stabilization and synapse formation.

Key words: development; dendritic spine; filopodium; glutamate receptor; NMDA receptor; $P S D-95$
The postsynaptic density (PSD) protein PSD-95 is a founding member of the growing superfamily of PDZ (PSD-95-Disks large-zona occludens1/2) domain-containing proteins (Craven and Bredt, 1998; Fanning and Anderson, 1999; Garner et al., 2000). The shared structural feature of these proteins, the PDZ domain, is made up of a repetitive sequence motif of $\sim 90$ amino acids that binds almost exclusively to $\mathrm{C}$-terminal consensus sequences of target proteins. Some PDZ proteins [e.g., SAP90/ PSD-95 (synapse-associated protein-90/PSD-95) and CASK (calcium calmodulin-dependent serine kinase)] contain additional protein-protein interaction sites, such as Src homology 3 (SH3) and/or guanylate kinase (GK) domains, whereas other PDZ proteins [e.g., GRIP/ABP (glutamate receptor-interacting protein/ AMPA-binding protein) and PICK-1 (protein interacting with C-kinase-1)] lack these (Scannevin and Huganir, 2000). Common to most PDZ-proteins is their function in the structural and functional organization of signaling complexes at presynaptic and postsynaptic elements through protein-protein interactions (Garner et al., 2000; Scannevin and Huganir, 2000).

PSD-95 is an integral protein of the postsynaptic density at excitatory synapses. Binding partners of PSD-95 include the NMDA (via PDZ1 and PDZ2) and kainate (via SH3) receptors and the cell adhesion molecule neuroligin (via PDZ3). Furthermore, binding to the proteins CRIPT [ (cysteine-rich interactor of PDZ three) via PDZ3] and GKAP [(guanylate kinase domain-

\footnotetext{
Received Aug. 8, 2001; revised Aug. 8, 2001; accepted Sept. 11, 2001.

T.H.M. is supported by an operating grant from the Canadian Institutes for Health Research (CIHR) and is a CIHR Scientist. O.P. is supported by a University Graduate Fellowship. We thank A. E. El-Husseini and D. S. Bredt for providing GFP-tagged PSD-95 constructs and antibodies.

Correspondence should be addressed to Dr. Tim Murphy, Kinsmen Laboratory, Department of Psychiatry, University of British Columbia, 2255 Wesbrook Mall, Detwiller Pavilion Room 4N1, Vancouver, British Columbia V6T 1Z3, Canada. E-mail: thmurphy@interchange.ubc.ca.

Copyright (C) 2001 Society for Neuroscience $\quad 0270-6474 / 01 / 219325-09 \$ 15.00 / 0$
}

associated protein) via GK] can tether PSD-95 and its binding partners to the intracellular tubulin and actin lattice, respectively (Scannevin and Huganir, 2000). PSD-95 exhibits a clustered distribution that is dependent on palmitoylation of two cysteine residues in the 3 and $5 \mathrm{~N}$-terminal positions of the protein (Craven et al., 1999). Studies in neuronal cell cultures have shown that, during early postnatal development (between 1 and 3 weeks in vitro), PSD-95 clustering shifts from a mostly nonsynaptic to a mostly synaptic pattern of localization (Rao et al., 1998). It has been suggested that this shift is associated with formation and possibly consolidation of early synaptic contacts (Rao et al., 1998; Garner et al., 2000). Formation of synaptic contacts may be facilitated by dendritic filopodia, highly motile and actin-rich protrusions that render dendritic branches during this early developmental period (Harris, 1999; Jontes and Smith, 2000). As a number of imaging studies have shown, filopodial motility significantly decreases during the first 3 weeks in vitro (Dailey and Smith, 1996; Ziv and Smith, 1996; Dunaevsky et al., 1999) and in vivo (Lendvai et al., 2000), paralleling the progress in synaptic localization of PSD-95 (Jontes and Smith, 2000). A current hypothesis is that this process of stabilization underlies the conversion of filopodia into more mature spines (Jontes and Smith, 2000; Parnass et al., 2000).

Previous evidence for a role of filopodia in synaptic contact formation (Ziv and Smith, 1996; Fiala et al., 1998) has been substantiated recently by an imaging study demonstrating that induction of functional presynaptic boutons can be rapid, within $\sim 45$ min after filopodia have initiated axo-dendritic contact (Friedman et al., 2000). This rapid timescale requires mechanisms to quickly target and establish the complex protein arrays in both developing presynaptic and postsynaptic compartments. Presynaptically, this process may be promoted by shuttling prepackaged active zone protein complexes into developing boutons (Ahmari et al., 2000). However, if analogous mechanisms exist 
for the rapid establishment of postsynaptic protein arrays is still unknown.

Here we use simultaneous time-lapse imaging of green fluorescent protein (GFP)-labeled PSD-95 and of filopodial motility to examine the disposition of PSD-95 clusters in dynamic filopodia. We report that PSD-95 clusters are transported as modular units and become associated with stable filopodia.

\section{MATERIALS AND METHODS}

Cell culture and particle-mediated gene transfer. Embryonic cortical neurons and glia were dissociated from 18-d-old Wistar rat fetuses and cultured on poly-D-lysine-coated $12 \mathrm{~mm}$ glass coverslips at a density of $\sim 1.5$ million cells per milliliter (Mackenzie et al., 1996). Cells were kept for 6-18 d in vitro (DIV) before use in experiments. Constructs encoding the following proteins were used: (1) wild-type (WT) full-length PSD-95; (2) full-length mutant (MUT) PSD-95/GFP in which the cysteine residues in the 3 and $5 \mathrm{~N}$-terminal positions were changed to serine residues (cys3,5ser) (Craven et al., 1999); and (3) GFP (Green Lantern, Life Technologies, MD) alone. PSD-95 constructs were generated by PCR and subcloned into the HindIII and EcoRI of GW1 (British Biotechnology) (Topinka and Bredt, 1998); GFP was subcloned in-frame at the C terminus of PSD-95 at the EcoRI site. For particle-mediated biolistic gene transfer, cDNA constructs were ligated onto $0.6 \mu \mathrm{m}$ gold microcarriers at a ratio of $1 \mu \mathrm{g}$ of DNA/1 mg of gold. Biolistic gene transfer was performed on cell cultures at 3-6 (average, $3.5 \pm 0.2$ ) DIV using a Bio-Rad (Herts, UK) Helios gene gun with helium pressure adjusted to140-160 psi and cultured for at least 3 more days after transfection. For experiments, PSD-95/GFP-expressing cells were sorted into two in vitro age groups. The first group consisted of cells at an average age of $7.9 \pm$ 0.3 DIV (range, $6-10$ DIV; $n=26$ cells); the second group of cells were cultured for $14.2 \pm 0.4$ DIV on average (range, $12-18$ DIV; $n=15$ cells). For simplicity, we refer to the young and old cultures by their average in vitro age (8 and 14 DIV, respectively).

Imaging and electrophysiology. Coverslips were placed into a customized perfusion chamber $(\sim 500 \mu \mathrm{l} \mathrm{vol})$ and fixed by platinum weights to prevent movement. The cultures were continuously perfused $(\sim 1.5 \mathrm{ml} /$ min) with an HBSS containing (in mM): $137 \mathrm{NaCl}, 2.5 \mathrm{CaCl}_{2}, 1.0 \mathrm{MgSO}_{4}$, $5.0 \mathrm{KCl}, 0.34 \mathrm{Na}_{2} \mathrm{HPO}_{4}\left(7 \mathrm{H}_{2} \mathrm{O}\right), 10.0 \mathrm{Na}^{+}$-HEPES, $1.0 \mathrm{NaHCO}_{3}$, and 22.0 glucose at $\mathrm{pH} 7.4(\sim 305 \mathrm{mOsm})$. The solution was heated to $32^{\circ} \mathrm{C}$ using a flow-through heater (Warner Instruments, Hamden, CT). Wholecell patch clamp on GFP fluorescence-positive cells was conducted using an Axon Instruments (Foster City, CA) Axopatch 200B amplifier and $7-10 \mathrm{M} \Omega$ electrodes pulled from $1.5 \mathrm{~mm}$ glass capillaries. The patch pipette solution contained $122 \mathrm{mM} \mathrm{K}^{+}$-gluconate, $20 \mathrm{~mm} \mathrm{NaCl}, 5 \mathrm{~mm}$ $\mathrm{Mg}^{2+}$-ATP, $0.3 \mathrm{~mm}$ GTP, $10 \mathrm{~mm}$ HEPES, $100 \mu \mathrm{M}$ EGTA, and $500 \mu \mathrm{M}$ sulforhodamine 101 (Molecular Probes, Eugene, OR), pH 7.2. The solution was allowed to perfuse into cells during whole-cell recording for 3-5 min, during which spontaneous postsynaptic currents were recorded. After that period, the electrode was carefully removed from the cell soma, allowing the membrane to reseal. The dye was allowed to equilibrate in the cell for at least $10 \mathrm{~min}$ before imaging experiments were performed.

Confocal time-lapse microscopy was performed on a Bio-Rad MRC 600 system attached to a Olympus Optical (Tokyo, Japan) BX50WI upright microscope using an Olympus Optical 0.9 numerical aperture $60 \times$ water immersion objective. The confocal pinhole was set to 3-4 (Bio-Rad units), and two-channel imaging was performed in slow scan mode (without averaging). For data acquisition, every 5-9 min (average, $7 \pm 2 \mathrm{~min}$ ), a (vertical) $Z$-series was taken over an area of $96 \times 128 \mu \mathrm{m}$ $(384 \times 512$ pixels $)$ containing the full extent of the dendrite of interest. The spacing of successive $Z$-images was $1 \mu \mathrm{m}$ and provided sufficient overlap between sections as the $Z$-resolution of the imaging system at the settings used was $2.5 \mu \mathrm{m}$ (measured as the width of the point spread function at half-maximum fluorescence intensity). The number of $Z$-images acquired ranged from three to seven but was always constant within an experiment. To correct for potential vertical movement of the specimen between time points, all $Z$-series in one experiment were started from a marker point chosen within the first optical plane.

Immunohistochemistry. For quantifying colocalization of PSD-95 clusters with presynaptic markers, cell cultures expressing WT PSD-95/GFP were fixed in paraformaldehyde at either 8 or 14 DIV. Cell cultures were immunolabeled using a mouse ( $\operatorname{IgG})$ monoclonal antibody $(\mathrm{Ab})$ against synaptophysin (Boehringer Mannheim, Mannheim, Germany) at 28 $\mu \mathrm{g} / \mathrm{ml}(1: 50)$ and an $\mathrm{IgG}$ polyclonal donkey-derived Texas Redconjugated secondary Ab (Jackson ImmunoResearch, West Grove, PA)

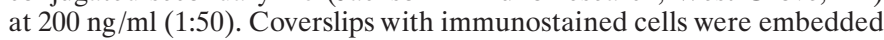
in anti-fade (Molecular Probes) agent, and confocal dual-channel imaging was performed using a $100 \times$ Zeiss (Oberkochen, Germany) Fluar oil-immersion lens.

Analysis. For analysis of PSD-95 localization and filopodial stability, only cells were used that did not show any morphological signs of cellular degeneration or impending death (i.e., cell swelling and/or collective loss of filopodia) before, during, or after imaging. For each time point, fluorescence data collected in a $Z$-series was projected into one layer using the maximum intensity method. Two-channel confocal images were separated off-line into their respective channels (sulforhodamine and GFP signal) and coded. This procedure allowed analysis of filopodial-spine stability to be performed on the sulforhodamine signal (red channel) without knowledge of which construct (WT PSD-95 or MUT PSD-95) the cells were transfected with. To be included for analysis, a filopodium-spine had to fulfill the following criteria: (1) being $>1$ and $<10 \mu \mathrm{m}$ in length, (2) not contacting another process, (3) not being extensively branched ( $<3$ branches), and (4) being sufficiently filled with dye so that it is clearly visible throughout the entire period of imaging $(\sim 1 \mathrm{hr})$. Processes that fit these criteria were not further subcategorized into either filopodia or spines, because unambiguous distinction by solely morphological criteria cannot be always made. The stability of each filopodium was judged by evaluating its status over $1 \mathrm{hr}$ of imaging. A filopodium-spine was defined as stable if it was present (visible) during the whole course of image acquisition. An unstable filopodium-spine either appeared new and/or disappeared during the time course of imaging. After this analysis, for each cell, the $Z$-projection of the corresponding GFP signal (green channel) was superimposed on the sulforhodamine (red channel) signal.

For WT PSD-95/GFP-expressing cells, PSD-95 clustering was based on the data collected on the green channel. In spines and dendritic shafts, the characteristics of each cluster were assessed throughout the period of imaging. In cases in which clustering could not be unambiguously attributed to a specific accumulation of PSD-95/GFP rather than a volumetric effect of the dendritic process, the fluorescence intensity of a potential PSD-95/GFP cluster was divided by the fluorescence signal of an area adjacent to the cluster. This ratio was then compared with the fluorescence ratio between the same areas (obtained using the same coordinates) on the sulforhodamine (red) channel, which represents a volumetric marker. If the ratio obtained on the green channel was more than two times the ratio on the red channel, then the PSD/95-GFP clustering was concluded to be specific.

For analysis of colocalization between the Texas Red-conjugated Ablabeled presynaptic marker synaptophysin (red channel) and PSD-95/ GFP clusters (green channel), $Z$-series from both channels were superimposed. Synaptophysin and PSD-95/GFP clusters were considered colocalized and indicative of a putative synapse if they were present within the same focal plane and were overlapping by at least $0.25 \mu \mathrm{m}(1$ pixel row).

For statistical analysis, the nonparametric two-tailed Mann-Whitney and Kruskal-Wallis ANOVA tests were used, unless otherwise noted. Data are expressed as the mean \pm SEM.

\section{RESULTS}

We used transfection of GFP-tagged PSD-95 constructs to examine the dynamics of filopodia and spines during development of primary cortical neurons. Biolistic particle-mediated transfer of DNA constructs was performed at 3-6 (average, $3.5 \pm 0.2 ; n=41$ cells) $d$ after plating cell cultures. At this time, the total amount of endogenous PSD-95 and the degree of synaptic PSD-95 clustering is low (Rao et al., 1998). Hence, in transfected cells, GFP-tagged PSD-95 could exert its effect during the earliest stages of synaptic development. Transfection efficiency was generally low $(<1.0 \%)$ using the biolistic gene transfer method. However, single transfected neurons could easily be identified by their strong GFP fluorescence (Fig. 1). Using polyclonal antibodies to PSD-95, we confirmed previous findings (El-Husseini et al., 2000) demonstrating that PSD-95 was overexpressed by 5- to 10 -fold in cells transfected with PSD-95/GFP constructs when compared with untransfected neighboring cells (data not shown). 

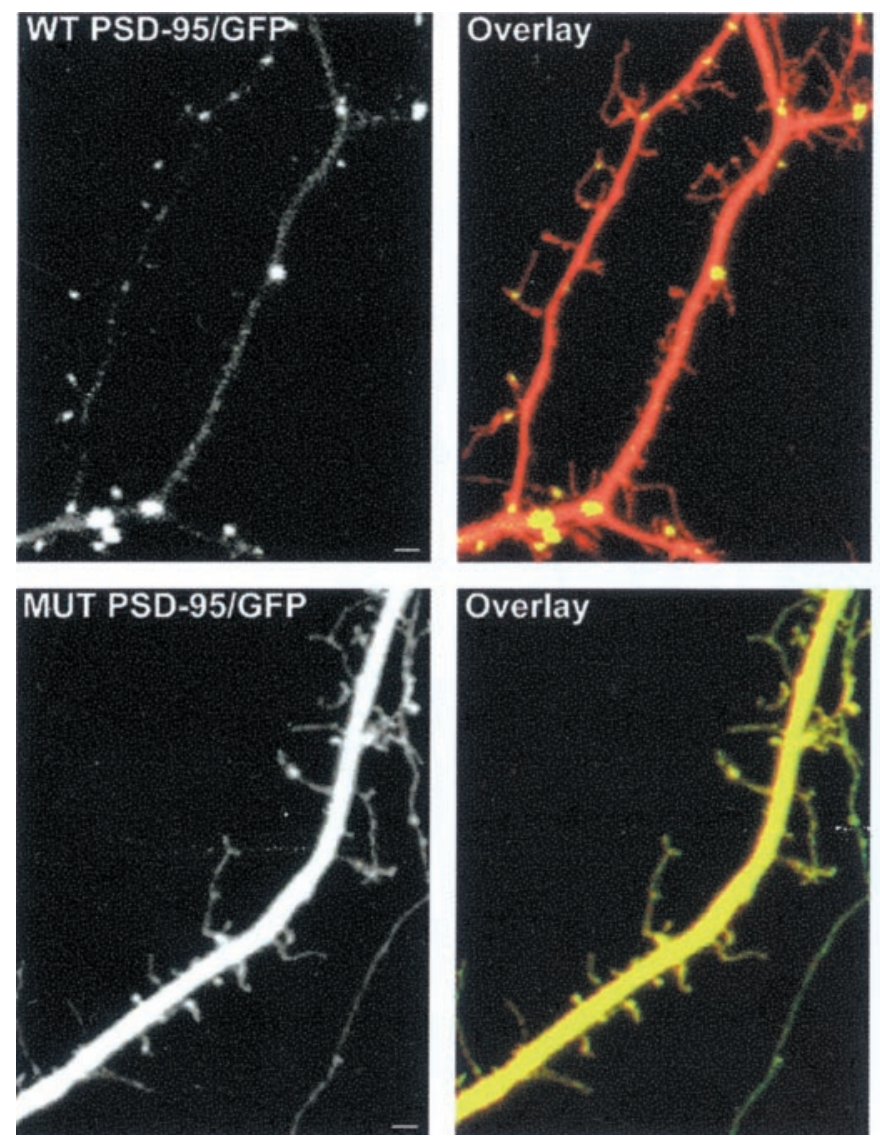

To assess the potential role of PSD-95 clustering in synapse development, maturation, and motility, we also transfected a mutant (palmitoylation deficient) nonclustering form of PSD-95 into sister cultures of cortical neurons. As shown previously (Craven et al., 1999), we find that the cys3,5ser MUT PSD-95 is expressed diffusely throughout the neuronal soma and processes compared with the punctate distribution pattern observed in cells transfected with the GFP-tagged WT form of PSD-95 (Fig. 1).

To simultaneously assess the localization of PSD-95 and the stability of filopodial processes, we used patch-clamp techniques to inject a second fluorescent marker (sulforhodamine) with different spectral properties into neurons expressing PSD-95/ GFP. This allowed us to reliably resolve filopodia and spines, including ones that do not express the PSD-95/GFP protein (Fig. 1, top row). At this early developmental stage, it was often not possible to further subcategorize processes into either filopodia or spines based solely on morphological features. Hence, we used the term filopodia-spines to describe all dendritic protrusions of length $>1$ and $<10 \mu \mathrm{m}$ (see also Materials and Methods). Using two-channel confocal microscopy, double-labeled cells were imaged on average every $7 \mathrm{~min}$ over a time period of 60-65 $\mathrm{min}$ (Fig. 2). Filopodia-spines with PSD-95/GFP clusters were stable (did not appear or retract) over the entire period of imaging. Time-lapse imaging indicated that, although the PSD-95/GFP clusters themselves were stable, the unlabeled membranes around the cluster were in constant motion (Fig. 2). Furthermore, nearby unlabeled (by PSD-95/GFP) processes were found to transiently extend and retract (Fig. 2) (see animation for Fig. 2 at www.interchg.ubc.ca/neurosci/faculty/murphy.html\#j_ns2).

To remove potential experimenter bias, two-color (PSD-95/ GFP and sulforhodamine) fluorescence images were separated
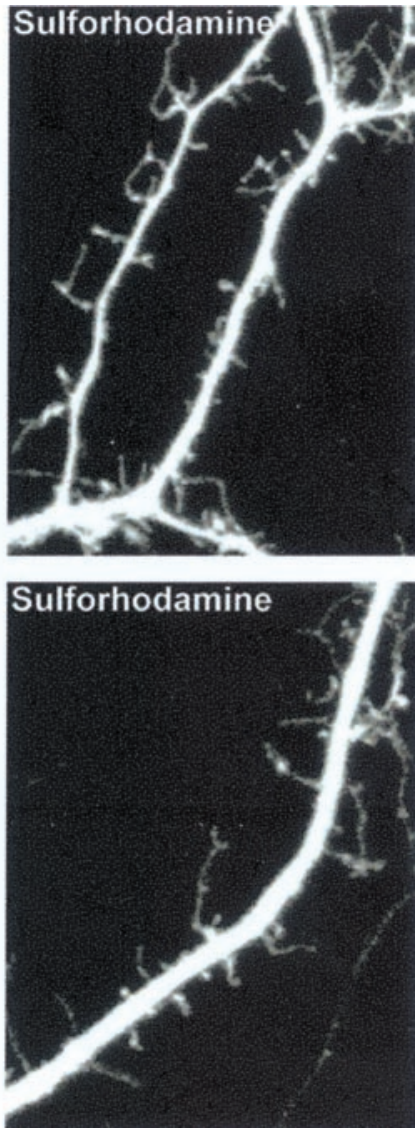

Figure 1. Two-channel confocal imaging to independently analyze PSD-95/ GFP distribution and dendritic morphology. Cultured cortical neurons were transfected with GFP-tagged WT (top row) or cys3,5ser MUT (bottom row) forms of PSD-95. Before imaging, cells were colabeled with sulforhodamine. Dual-channel confocal imaging was performed, allowing the separation of the GFP (left panels) and sulforhodamine (right panels) signal. The middle panels show pseudocolored composite images spective row; areas of GFP (green) and sulforhodamine (red) colocalization appear yellow. Localization of PSD-95/ GFP in dendritic shafts and spines is clustered in cells expressing WT PSD95/GFP (top row) and dispersed in cells expressing the MUT PSD-95/GFP form (bottom row). Cells were cultured for $13 \mathrm{~d}$ (top row) and $8 \mathrm{~d}$ (bottom row). Scale bar, $5 \mu \mathrm{m}$. of the left and right panels in their re-

into their respective channels and coded. Analysis of filopodiaspine turnover was performed using only the sulforhodamine (red) channel. This procedure ensured that analysis of filopodiaspine movement was done without previous knowledge of PSD-95 disposition for particular spines or the type of construct transfected. To examine the dynamics of PSD-95 clustering and its potential role during early synaptic development, we compared PSD-95 translocation and filopodium - spine motility at two different in vitro ages. Cells from the first group (labeled 8 DIV) were studied at an average age of $7.9 \pm 0.3$ DIV (range, 6-10 DIV; $n=26$ cells), a period that is expected to correspond to the early onset phase of synaptogenesis (Lee and Sheng, 2000). The second group (labeled 14 DIV) consisted of cells at, on average, $14.3 \pm 0.5$ DIV (range, $12-18$ DIV; $n=15$ cells), a developmental stage characterized by ongoing synapse formation ( $\mathrm{Ziv}$ and Smith, 1996), stabilization of newly formed spines (Lee and Sheng, 2000), and enrichment of synaptic proteins within these structures (Rao et al., 1998). First, we compared the density of filopodia-spines between the age groups. To do this, we normalized the number of protrusions to the length of dendrite imaged for each neuron. In neurons overexpressing WT PSD-95/GFP, we found a significant increase (by $37 \%$; $p<0.05$ ) in the total number of filopodia-spines between cells at 8 and 14 DIV. There was a disproportionally large increase (by $135 \%$; $p<0.001$ ) in filopodia-spines that were stable (during $1 \mathrm{hr}$ of time-lapse imaging) and a concomitant reduction (by 65\%; $p<0.01$ ) of unstable filopodia-spines (Fig. 3A). This developmental stabilization between 8 and 14 DIV was reflected by a significant increase (from $14 \pm 3$ to $36 \pm 6 \% ; p<0.004$ ) in the proportion of filopodia-spines that associated with PSD-95/GFP clusters. Similarly, for cells transfected with MUT PSD-95/GFP, we found that 

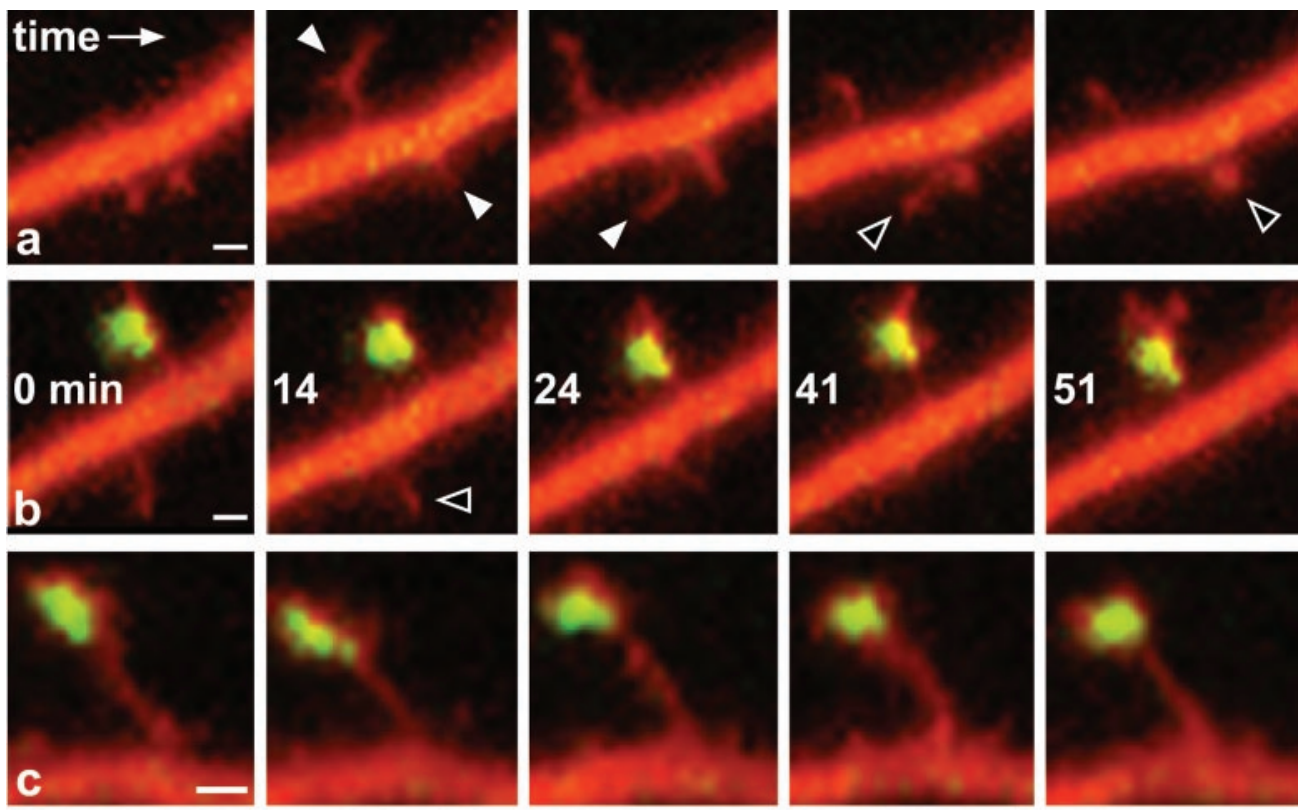

Figure 2. Confocal time-lapse analysis of filopodia-spine stability and PSD-95 clustering. Time-lapse series of a neuron $(10 \mathrm{~d}$ in vitro $)$ that expressed WT PSD95/GFP ( green) and was filled with sulforhodamine (red). Spots of PSD-95/ GFP clustering appear yellow. The bottom panel shows an overview of the dendrite. Scale bar, $5 \mu \mathrm{m}$. Three areas are magnified (see dashed outlines labeled $a-c$ ). Time series in the top rows advances in columns from left to right for the three areas of interest; the second row shows time stamps in minutes. For simplicity, not every time point is shown. Newly forming (white arrowheads) and disappearing (black arrowheads) filopodia-spines are marked. Note that PSD-95/GFP-containing structures are stable over time (i.e., they do not turnover) but can exhibit structural changes. Scale bars: magnified areas, $1 \mu \mathrm{m}$; bottom panel overview, $5 \mu \mathrm{m}$.

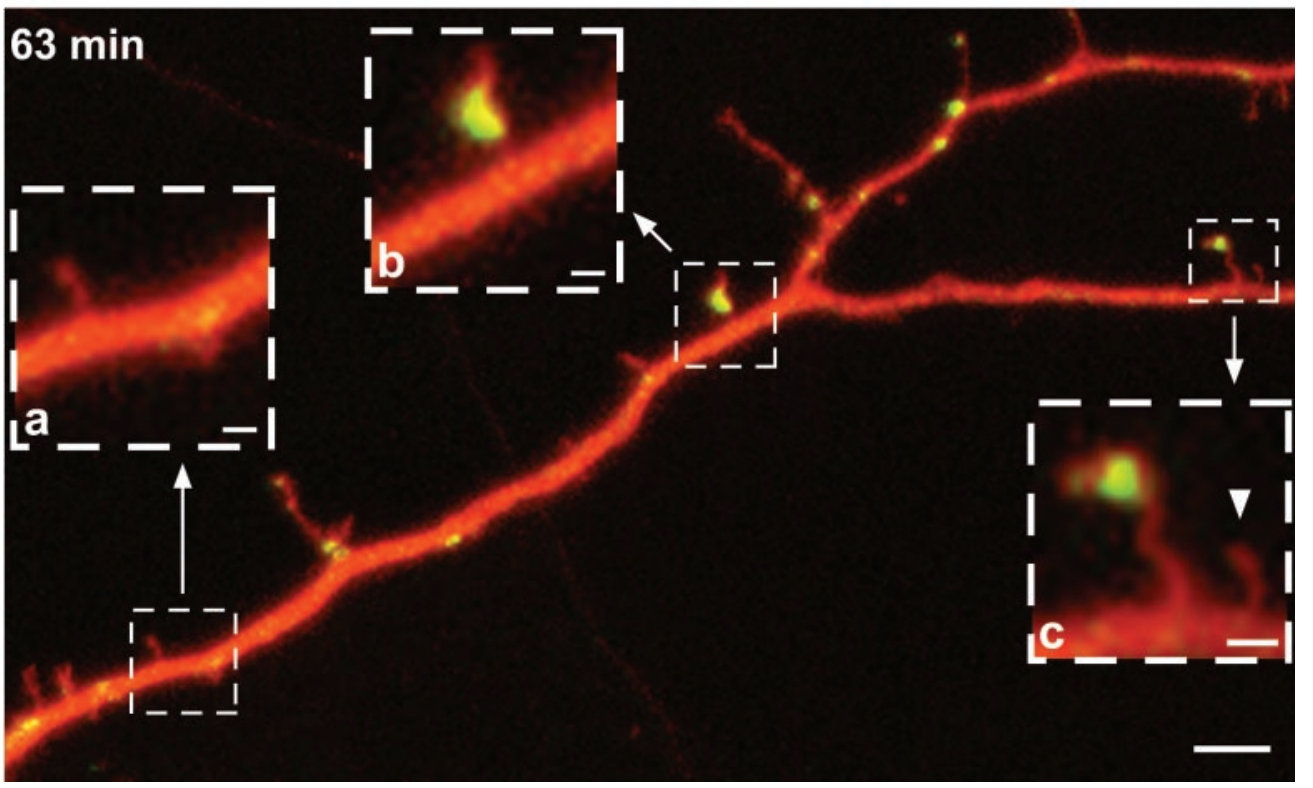

between 8 DIV ( $n=13$ cells) and 14 DIV ( $n=5$ cells $)$ the total number of filopodia-spines per millimeter increased by $22 \%$, the number of stable spines increased by $73 \%$, and the number of unstable filopodia-spines decreased by $56 \%$ (not significantly different from wild-type PSD-95/GFP data).

To reduce potential bias associated with the selection of particular segments of dendrite for imaging, we normalized the number of stable and unstable filopodia-spines to the total number of filopodia-spines per cell. Using this procedure, we find no difference in the proportions of stable and unstable filopodiaspines between MUT and WT PSD-95/GFP-expressing cells in either age group ( $8 \mathrm{DIV}, p>0.2 ; 14 \mathrm{DIV}, p>0.5$ ). Additionally, there was no difference in the proportion of stable and unstable filopodia-spines between PSD-95/GFP-expressing cells and control cells $(n=9)$ expressing only GFP in either age group (8 DIV, $p>0.5$; 14 DIV, $p>0.7$; Kruskall-Wallis test).

To evaluate the relationship between PSD-95 clustering and filopodia-spine dynamics in neurons overexpressing the WT
PSD-95/GFP construct, we separately analyzed data from the PSD-95/GFP (green) channel. First, we found a significant increase in the overall density of PSD-95 clusters in dendrites (from $86 \pm 9$ to $117 \pm 19$ per millimeter of dendrite; $p<0.05)$ when comparing between stages of earlier (8 DIV) and later (14 DIV) in vitro development. This difference was primarily attributable to a significant increase of filopodia-spines possessing PSD-95/GFP clusters (from $17 \pm 4$ to $60 \pm 12$ per millimeter of dendrite; $p<$ 0.001) (Fig. 3B). However, the density of PSD-95/GFP clusters in dendritic shafts did not change significantly over the same period (from $69 \pm 8$ to $57 \pm 9$ per millimeter of dendrite; $p>0.4$ ) (Fig. $3 B)$. To determine whether the increasing number of PSD-95/ GFP clusters was attributable to an increase in the cluster fluorescence intensity (and therefore in the likelihood of detection), we compared the PSD-95/GFP fluorescence signal between 8 DIV ( $n=6$ cells) and 14 DIV ( $n=6$ cells) using identical imaging settings. Our results indicate that this was not the case because we find that the intensity of PSD-95/GFP clusters actually 

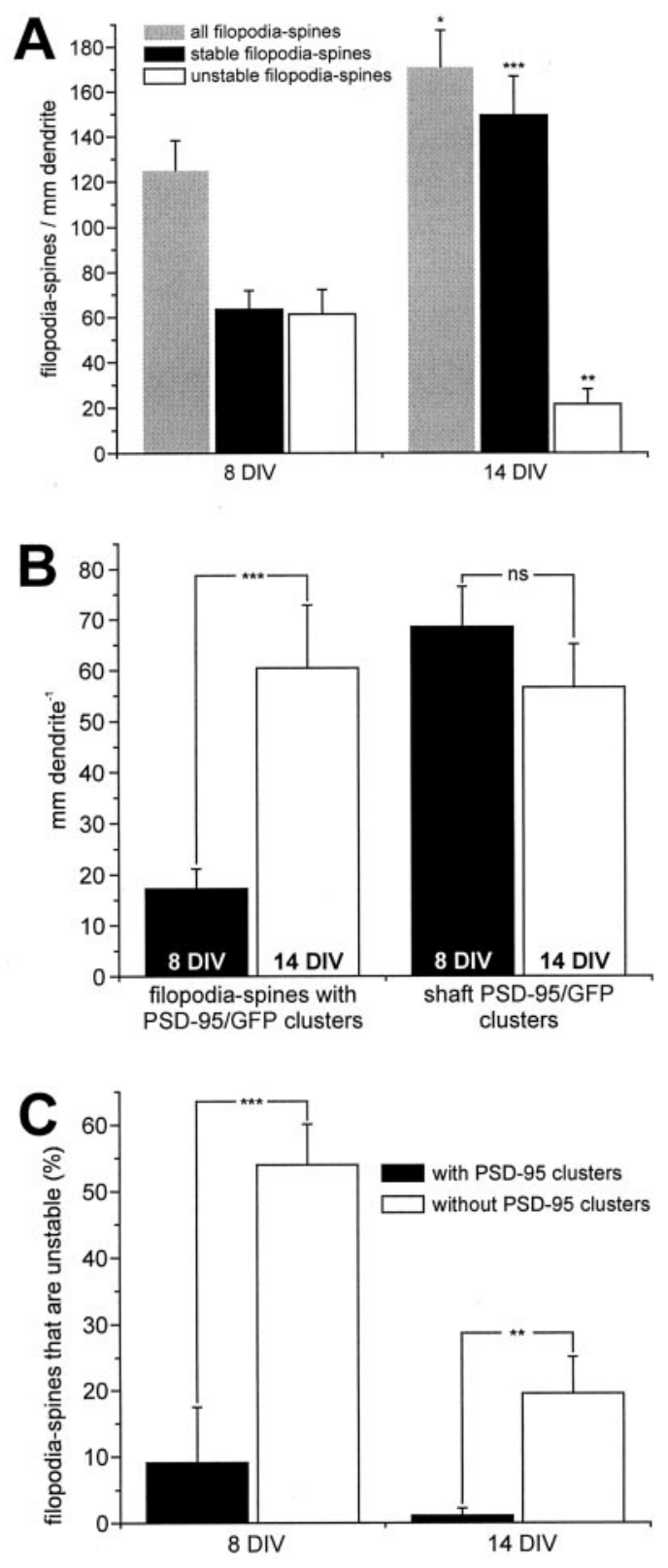

Figure 3. Quantitation of filopodia-spine development and the association with PSD-95/GFP clusters. $A$, Increase in number and stability of filopodia-spines during early neuronal development. Cells were cultured for, on average, either 8 or $14 \mathrm{~d}$, and filopodia-spine dynamics were imaged using confocal time-lapse microscopy over $1 \mathrm{hr}$. The total number of filopodia-spines increases (by 37\%; * $p<0.05$ ) between the two age groups. A disproportionately large increase in the number of stable filopodia-spines (by $135 \%$; *** $p<0.001$ ) was observed. A concomitant decrease (by $65 \%$; * $p<0.01$ ) in the number of unstable filopodia-spines was observed. All numbers are normalized to dendritic length. $B$, Increase in the number of filopodia-spines with PSD-95 clusters but not of PSD-95 shaft clusters during early neuronal development. Comparison of cells in the younger ( $n=13$ cells) with the older $(n=10$ cells $)$ age group indicated a significant increase in the density of filopodia-spines with PSD-95/GFP clusters (by 350\%; *** $p<0.001$ ). No significant (ns) change in the number of PSD-95 shaft clusters $(21 \%$ decrease; $p>0.4)$. $C$, Filopodia-spines with PSD-95/GFP clusters are more stable than filopodia-spines without clusters. The stability of filopodia-spines was compared between filopodia-spines with PSD-95/GFP clusters and filopodia-spines devoid of clusters. Filopodia-spines without PSD-95/GFP clusters are significantly more unstable at $8 \mathrm{DIV}\left({ }^{* * *} p<0.001\right)$ and $14 \mathrm{DIV}\left({ }^{* *} p<0.005\right)$ compared with filopodia-spines with PSD-95/GFP clusters (8 DIV: $n=13$ neurons, 303 filopodia-spines; 14 DIV: $n=10$ neurons, 440 filopodia-spines). decreases (by $52 \%$; $p<0.001$ ) between 8 and 14 DIV, confirming reports of declining levels of recombinant protein expression over this time (Craven et al., 1999).

In young neurons, only a subset of filopodia-spines (14\%) contain PSD-95/GFP clusters (Fig. 3), providing a scenario to test the relationship between the presence of clusters and stability. Accordingly, we analyzed the stability of filopodia-spines with PSD-95/GFP clusters in WT PSD-95/GFP-expressing cells $(n=$ 23). We find that, of 190 filopodia-spines that possessed PSD-95/ GFP clusters, only $3(1.6 \%)$ were unstable during the $\sim 1 \mathrm{hr}$ period of imaging (Fig. 3C). This high level of stability differed significantly from that of filopodia-spines without PSD-95/GFP clusters in both age groups. At 8 DIV, $54 \pm 6 \%$ of all filopodiaspines without PSD-95/GFP clusters were unstable, whereas only $9 \pm 8 \%$ with PSD-95/GFP clusters were $(p<0.001)$ (Fig. $3 C)$. At 14 DIV, only $1 \pm 1 \%$ of all filopodia-spines with PSD-95/GFP were unstable versus $20 \pm 6 \%$ without PSD-95/GFP clusters $(p<$ 0.005) (Fig. 3C).

By restricting our acquisition of data to a $1 \mathrm{hr}$ period, we minimized potential damage attributable to phototoxicity. However, it is possible that by selecting a single arbitrary time point we may have underestimated the number of unstable spines. To further examine the relative stability (i.e., turnover time) of filopodia-spines, additional experiments $(n=3)$ were performed in which the total imaging time was increased to $2.5 \mathrm{hr}$ (sampling intervals extended to $15 \mathrm{~min}$ ). As expected, we find that, by increasing the imaging time from 1 to $2.5 \mathrm{hr}$, a significantly higher proportion of filopodia-spines become unstable ( $83 \pm 4$ vs $48 \pm$ $6 \%$ at $1 \mathrm{hr} ; p<0.05$; one-tailed Mann-Whitney test). However, we still find that all filopodia-spines $(100 \%)$ associated with PSD-95/GFP clusters ( $n=8 ; 11 \%$ of all filopodia-spines) are stable during the $2.5 \mathrm{hr}$ imaging period. Together, these data suggest that the average half-life time for filopodia-spine structures without PSD-95 clusters approximates $1 \mathrm{hr}$ and that most of these structures will eventually turnover during longer time periods. On the other hand, PSD-95/GFP-associated structures display structural stability over extended time periods.

Because our data indicate that filopodium-spine structures with associated PSD-95 clusters are highly stable, we next determined the stability of the PSD-95 clusters themselves. Of all PSD-95 clusters found in dendritic shafts $(n=317)$ and in filopodia-spines $(n=190)$, we did not find a single case demonstrating the breakdown (disappearance) and/or the appearance of the cluster within $1 \mathrm{hr}$ of time-lapse imaging, indicating a slow turnover rate for PSD-95 clusters. However, unidirectional or bidirectional lateral movement of PSD-95 clusters within dendritic shafts and filopodia-spines could be observed (Fig. 4A,B). Of all shaft PSD-95 clusters, $14 \pm 4 \%$ exhibited lateral movement at $8 \mathrm{DIV}$ over a $1 \mathrm{hr}$ period. This proportion decreased significantly to $2 \pm 1 \%$ at $14 \mathrm{DIV}(p<0.05)$. The range of movement of PSD-95 shaft clusters was usually relatively small (1-3 $\mu \mathrm{m})$ but could in single cases be up to $5 \mu \mathrm{m}$ within $20 \mathrm{~min}$ of imaging. Additionally, small 1-2 $\mu \mathrm{m}$ movements of PSD-95/GFP clusters could be observed in $20 \pm 9 \%$ (8 DIV) and $17 \pm 7 \%$ (14 DIV) of all filopodia-spines with PSD-95 clusters. Moreover, in a few occasions ( $<5 \%$ of all clusters), we were able to observe "splitting" of a PSD-95 cluster (Fig. 4A,B) or "merging" of two or more PSD-95 clusters into one. For more information, see on-line animated version of Figure 4 (www.interchg.ubc.ca/neurosci/ faculty/murphy.html\#j_ns4).

Although PSD-95 clusters in filopodium-spine structures were mostly stable, we did occasionally observe filopodia-spines or 

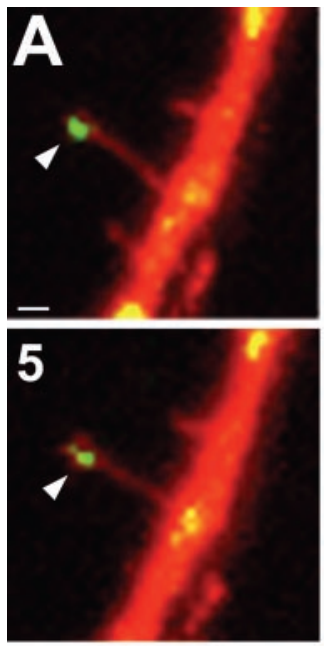

Figure 4. Dynamic properties of PSD-95 clusters within developing neurons. Partial time-lapse series of dendrites of cells transfected with WT PSD95/GFP (green) and filled with Areas of dye colocalization appear yellow. Images in respective columns were taken consecutively, with the exception of the last time point in $A-C$, which represents the last data point of the series. Time stamps are in minutes; all series start at $0 \mathrm{~min}$. Scale bars, $1 \mu \mathrm{m}$. $A$, A 10 DIV neuron in which a PSD-95 cluster (white arrowhead) moves out of a filopodium-spine. Spine splitting at 27 min into a two-headed structure. $B$, A 6 DIV neuron in which a shaft cluster splits into two domains (0-5 min; top, black arrowheads). A cluster (bottom) and 16 min time points; white arrowhead) and transiently splits (22 min; black arrowhead). Filopodium-spine consecutively undergoes rapid outgrowth $(22 \mathrm{~min})$ and retraction $(58$ min). $C$, An 8 DIV neuron in which filopodium-spine outgrowth (0-9 min; black arrowhead) precedes localization of cluster into its tip (14-19 min; white arrowhead) and additional elongation of the structure with arrested cluster in its tip (26-50 min). D, An 8 DIV neuron in which a small PSD-95/GFP-labeled structure reversibly protrudes from a large shaft cluster (top). Another small filopodium reversible protrudes (bottom) from the dendritic shaft, carrying a PSD-95 cluster from the dendritic shaft with it.
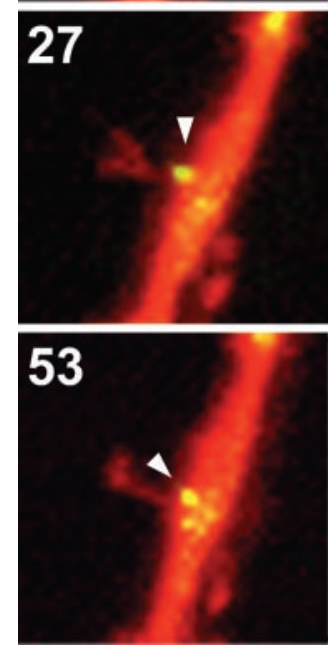
sulforhodamine $($ red $)$ to assess structure. leaves a filopodium-spine (between 11
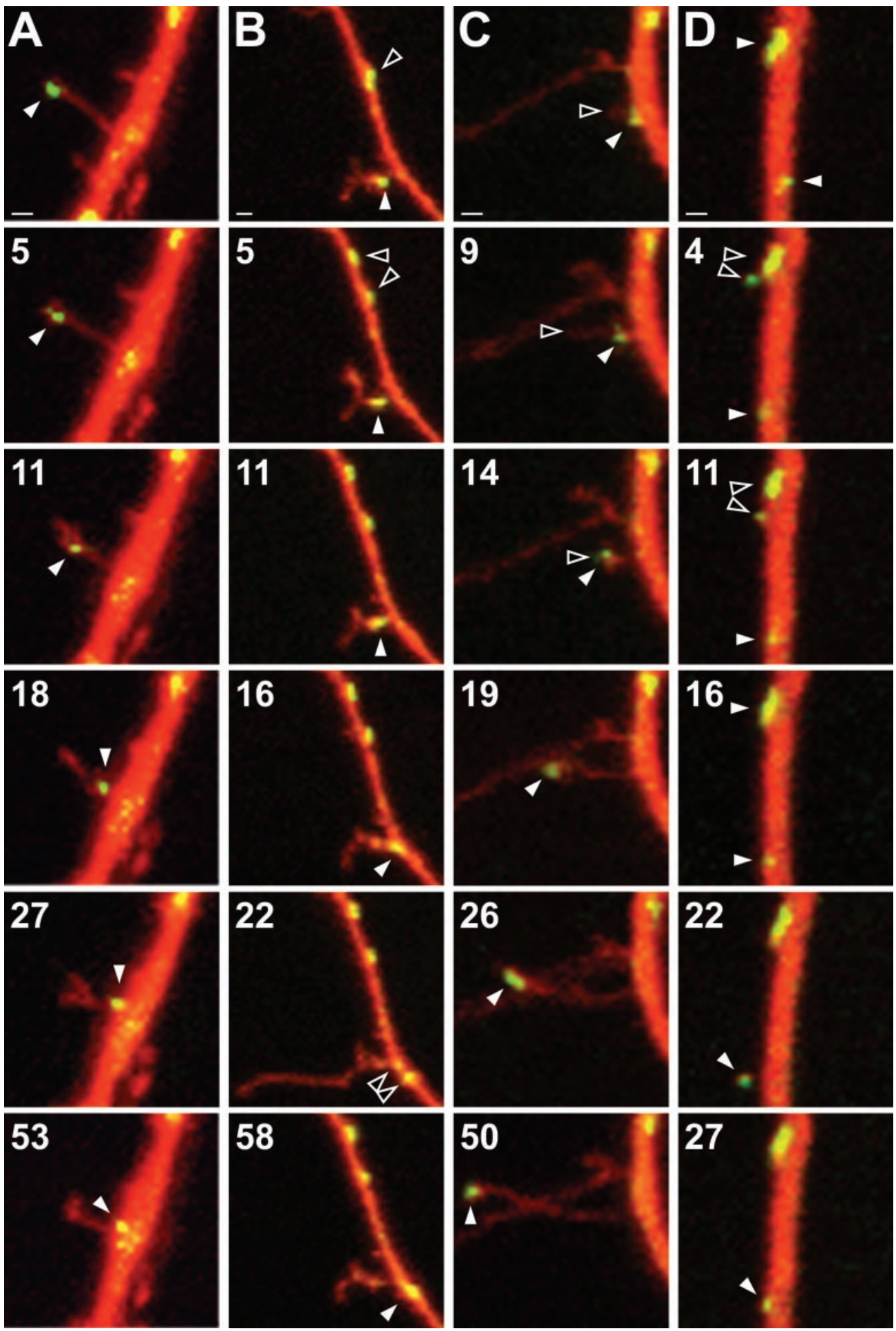

small precursors to these structures into which PSD-95/GFP clusters became transiently located (Fig. 4A,B). Interestingly, we found nine cases in which PSD-95 clusters either exited $(n=7)$ and/or entered a filopodium-spine $(n=2)$, usually within $<20$ min (Fig. 4). Seven of these cases were found in neurons within the younger age group (8 DIV), representing $13 \%$ of all filopodia-spines with PSD-95 clusters. In the older age group (14 DIV), we observed this phenomenon in only two cases (1\%) of filopodia-spines with PSD-95 clusters. Analysis of these images indicated that the PSD-95 clusters moved as modular units. Clusters were observed to move either independently along the length of a filopodium-spine (Fig. $4 A, B$ ) or to remain stationary and move as a consequence of a growing or retracting structure (Fig. $4 C, D$ ). In all cases in which PSD-95/GFP clusters were translocated, these processes appeared to occur rapidly (within minutes) and involved the coordinated relocation of an intact cluster rather than a gradual accumulation or breakdown of labeled material (see time courses in Fig. 4).

PSD-95/GFP movement within filopodia-spines was usually associated with significant structural changes of the structures 

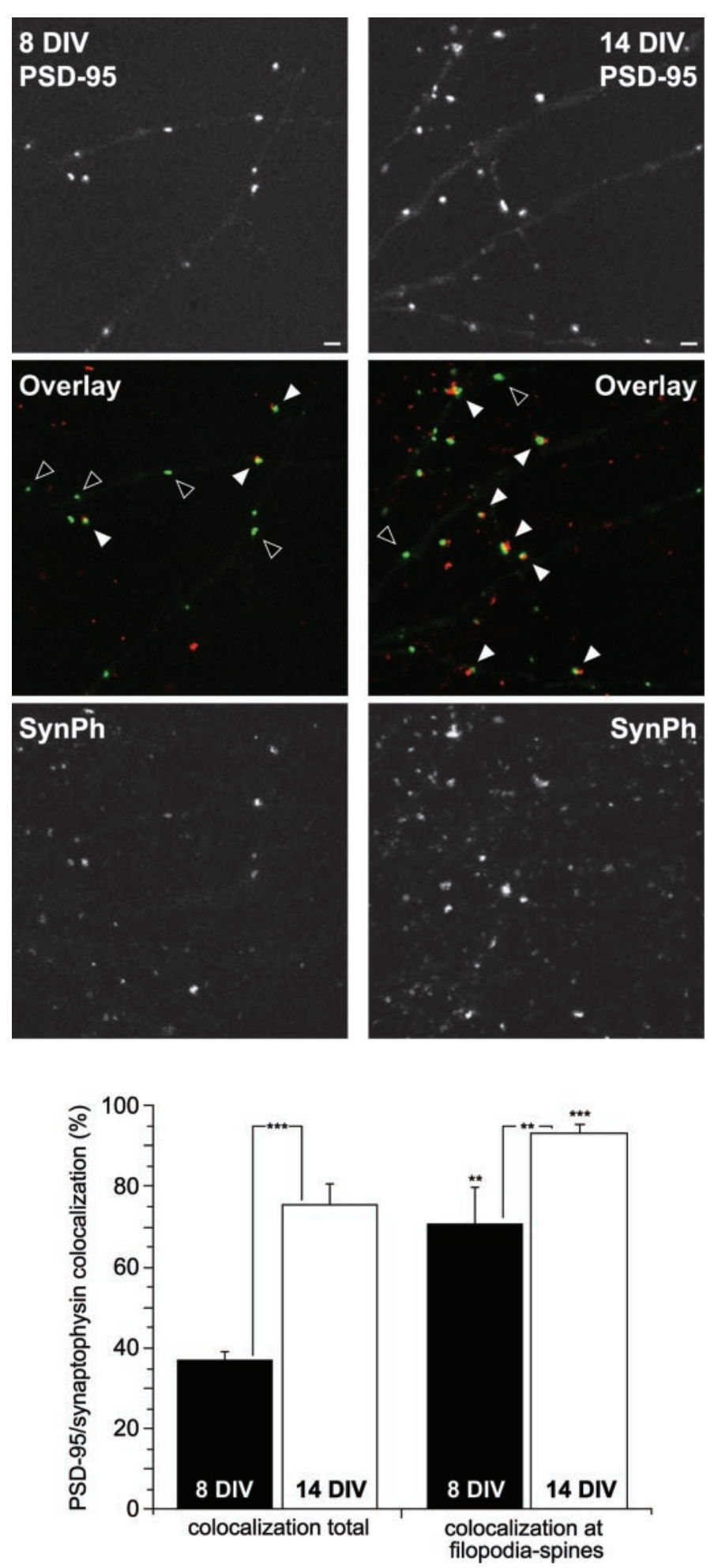

Figure 5. Developmental increase in the colocalization of PSD-95 and a presynaptic marker. Neurons were transfected with the WT PSD-95/GFP (top panels) construct and were immunostained for the presynaptic marker synaptophysin (SynPh; bottom panels) using a Texas Redconjugated secondary antibody. The left and right columns depict representative confocal images from dendritic regions immunostained at 8 and 14 DIV, respectively. Middle panels, Composites of the PSD-95 ( green) and synaptophysin (red) images. White arrows depict examples of PSD-95 and synaptophysin colocalization; black arrowheads indicate examples of PSD-95 clusters without corresponding synaptophysin clusters. Scale bar, $2 \mu \mathrm{m}$. Bar graph, Colocalization of PSD-95 and synaptophysin increases
(Fig. $4 A, B$ ). As illustrated in Figure $4 A$, retraction of a PSD-95 cluster from the tip of a filopodium-spine to the shaft occurred at a rate of $\sim 4 \mu \mathrm{m}$ in $30 \mathrm{~min}$ and was associated with a bifurcation of the filopodium-spine into a two-headed structure $(27 \mathrm{~min}$ image). In other cases, we observed that the translocation of PSD-95/GFP clusters was associated with a sudden outgrowth of the structure (Fig. 4B). Alternatively, PSD-95 shaft clusters could be "picked up" by outgrowing filopodia-spines (Fig. $4 C$ ) or shortlived proto-filopodial structures (Fig. $4 D$ ), and subsequently located into their growing tips (Fig. $4 C$ ). These data indicate the existence of two distinct processes: one for preassembling PSD-95 into clusters and one for subsequently localizing PSD-95 complexes into emerging filopodia-spines, which is reversible (see also animated material).

To assess whether the developmental changes in filopodiumspine stability and PSD-95 localization were accompanied by presynaptic changes, we used an immunostaining approach. A primary antibody against the presynaptic marker synaptophysin and a secondary Texas Red-conjugated antibody were used in cultures transfected with WT PSD-95/GFP at 8 and 14 DIV (Fig. $5)$. Analysis of colocalization between PSD-95/GFP and synaptophysin shows a significant increase $(p<0.0001)$ between 8 DIV (37 $\pm 2 \%$ of all PSD-95 clusters colocalizing with synaptophysin; $n=9$ cells $)$ and 14 DIV (75 $\pm 5 \% ; n=9$ cells) (Fig. 5). However, even at the younger age (8 DIV) when PSD-95/GFP was present in filopodia-spines (rather than dendrite shafts), colocalization with synaptophysin was significantly higher $(71 \pm 9 \% ; p<0.01)$ than the overall rate (Fig. 5). The same trend was apparent of older cultures (14 DIV) in which colocalization of PSD-95 and synaptophysin increased significantly to $93 \pm 2 \%(p<0.001)$ when only clusters in filopodia-spines were considered and shaft clusters were excluded (Fig. 5).

\section{DISCUSSION}

Dendritic filopodia are highly motile transient processes that move in an actin-dependent manner (Fischer et al., 1998). During early development, it is proposed that these processes continuously probe the extracellular environment for potential presynaptic partners (Saito et al., 1992; Dailey and Smith, 1996; Ziv and Smith, 1996; Fiala et al., 1998; Halpain, 2000). Recent data suggests a model for excitatory synapse development in which initial motile filopodial structures become stabilized in a partially activity-dependent manner, leading to the formation of mature dendritic spines (Jontes and Smith, 2000; Lee and Sheng, 2000; Segal, 2001). In fact, time-lapse experiments have indicated that a functional synapse can develop from a motile filopodium within $<1$ hr (Friedman et al., 2000). This observation has placed new time constraints on synaptogenesis and now focuses attention on the regulatory role that synaptic proteins play in this process (Jontes and Smith, 2000; Rao and Craig, 2000; Segal and Andersen, 2000). The integral postsynaptic density protein PSD-95 represents a conceivably good candidate protein for such a function because it can tether receptors and signal transduction proteins into complexes and cross-link them to structural protein

significantly between 8 DIV (37 $\pm 2 \%$ of all PSD-95 clusters are colocalized with synaptophysin; $n=9$ cells $)$ and 14 DIV $(75 \pm 5 \% ; n=9$ cells $)$ (left column). A significantly higher rate of colocalization was observed when PSD-95/GFP clusters were localized in filopodia-spines (compare the left and right columns for the same age group). $* * p<0.01 ; * * p<$ 0.001 
arrays using its protein-protein interaction domains (Allison et al., 1998, 2000; Zhang et al., 2001) (for review, see Garner et al., 2000; Scannevin and Huganir, 2000). Clustering of PSD-95 into filopodia and the consequent scaffolding of the postsynaptic signal transduction machinery may therefore provide an important step in the proper assembly of functional synapses.

Accordingly, we performed time-lapse imaging experiments with GFP-tagged PSD-95 to simultaneously monitor filopodialspine stability and PSD-95 localization during a time period of in vitro development believed to be critical for synaptogenesis and stabilization of dendritic processes. Although previous time-lapse studies of PSD-95 have been done (Okabe et al., 1999), they were performed on considerably older neurons that lacked filopodia. Furthermore, this study used relatively low-resolution wide-field microscopy and focused on PSD-95 dynamics over much longer time periods (up to $24 \mathrm{hr}$ ) with much lower sampling rates. We extended this work by using higher-resolution confocal imaging with considerably shorter time intervals $(5 \mathrm{~min})$. An additional advantage of our study was that we took multiple optical sections that spanned the vertical extent of the dendrite of interest to ensure that all filopodia-spine processes were indeed captured and that changes in process disposition did not reflect changes in the optical planes sampled. Finally, we introduced a second fluorescent marker into PSD-95/GFP-expressing neurons, which allowed us to label dendritic structure and analyze filopodialspine dynamics independently of PSD-95/GFP disposition. During the early developmental period of neurons used in the present study, it was often not to possible clearly classify a dendritic protrusion as either a filopodium or a spine. Hence, we grouped all dendritic protrusions matching the criteria outlined above (see Materials and Methods) into one category (filopodia-spines). This was done to prevent the introduction of an additional level of potential observer bias into our analysis. However, applying categorizing criteria for spines and filopodia established by others (Parnass et al., 2000; Sorra and Harris, 2000) most cautiously to our system, we can conservatively estimate that, even within the relatively older developmental group (14 DIV), $>50 \%$ of all protrusions show characteristics typical for filopodia, with lengths usually exceeding 2-3 $\mu \mathrm{m}$ and not with well defined heads (diameter not larger than that of the "neck") (Parnass et al., 2000; Sorra and Harris, 2000). However, many of these processes contained PSD-95 clusters (see Results). Within the younger group (8 DIV), the protrusions exhibit almost exclusively (>90\%) filopodial features and most were mostly devoid of PSD-95 clusters (see Results).

Consistent with previous data (Ziv and Smith, 1996; Fiala et al., 1998; Dunaevsky et al., 1999), we show that dendritic filopodia-spines are transient structures present during early development. Interestingly, recent data suggests that, in vivo, developing cortical neurons have active dendritic filopodia that extend and turnover with kinetics similar to what we reported (Lendvai et al., 2000). Furthermore, motile dendritic filopodia become considerably arrested at an in vitro age similar to their in vivo counterparts (Fiala et al., 1998; Dunaevsky et al., 1999), suggesting that important parallels between in vitro and in vivo development exist. Because overexpression of PSD-95 has been demonstrated to have a permissive role on spine development and transmitter release probability (El-Husseini et al., 2000), we overexpressed WT PSD-95 or MUT PSD-95, which lacks synaptic targeting and fails to form clusters, and determined whether correlates of this might be observed in developing filopodial-spine dynamics. We hypothesized that, if PSD-95 levels were limiting (in this system), we would be able to possibly drive the development of new filopodia-spines by overexpressing the wild-type protein and/or altering the dynamic development of existing ones. However, no significant change in either the number of filopodia-spines or their stability was observed when overexpression of either wildtype PSD-95/GFP or mutant (nonclustering) forms were compared. This result suggested that, under the conditions we used, either cluster formation of PSD-95 in filopodia-spines is not necessary for stabilizing these structures or endogenous PSD-95 cluster levels were sufficient to promote synapse development and stability. Therefore, it is likely that clustered PSD-95 is a marker for stable filopodia-spines but by itself is not always sufficient to induce stabilization. Alternatively longer periods of PSD-95/GFP expression may be required to promote development of mature spines. Previous reports showing a correlation between PSD-95 expression and spine maturation were from cell culture systems that were considerable older and more developed than the ones that we used (El-Husseini et al., 2000). Interestingly, these authors found that, in younger cultures (12 DIV), the spine number (as measured by the number of AMPA receptor clusters) was not altered in neurons transfected with PSD-95/GFP compared with wild-type controls (El-Husseini et al., 2000)

The fact that PSD-95/GFP overexpression does not affect spine development in our cultures importantly indicates that we were not perturbing the system and altering the properties or number of filopodia-spines that were studied with time-lapse imaging. It was under these conditions that, within WT PSD-95/GFPexpressing neurons, we observed a profound increase in the stability of filopodia-spines with PSD-95 clusters compared with filopodia-spines that lack PSD-95 clusters at both developmental stages studied. These findings suggest that events associated with the presence of clusters lead to the development of stable filopodia-spines, which, over longer time periods, may transform into functional postsynaptic spines with their typical morphological features (Lee and Sheng, 2000; Wong and Wong, 2000). At both developmental stages, only 14\% (8 DIV) and 36\% (14 DIV) of filopodia-spines associated with PSD-95/GFP clusters, indicating the relative immaturity of neurons during this period. Interestingly, we found that a considerable fraction of apparently stable filopodia-spines $(78 \pm 5$ and $59 \pm 6 \%$ for 8 and 14 DIV neurons, respectively) did not contain detectable PSD-95/GFP clusters. Spines lacking PSD-95 have also been reported by others (Friedman et al., 2000). One explanation for this finding is that we used a relatively short total imaging time $(\sim 1 \mathrm{hr})$ to quantify changes in filopodia-spine turnover. By restricting ourselves to this time period, we may therefore have underestimated the fraction of unstable filopodia-spines. Consistent with this proposal, we observe a significantly higher turnover rate of filopodia-spines when a longer imaging period $(2.5 \mathrm{hr}$ ) was used (data from $8 \mathrm{DIV}$ neurons; see Results).

Consistent with the relative stability of the PSD (Allison et al., 1998, 2000; Passafaro et al., 1999), we observe that clusters of PSD-95 were generally stable over a $1 \mathrm{hr}$ period. Although the PSD-95 clusters themselves were stable, we did observe a considerable number of clusters exhibiting at times substantial lateral movement over short periods of time $(\sim 1 \mu \mathrm{m} / \mathrm{min})$. Most interestingly, in a few cases, dendritic shaft PSD-95 clusters were able to move from the shaft or into a spine-filopodium or vice versa. In general, structures into which the cluster moved appeared to be quite stable. We proposed that the PSD-95 cluster might be moving with a group of associated postsynaptic proteins consistent with immunocytochemical and proteomic analysis data (Husi 
et al., 2000; Walikonis et al., 2000). Recently, it has been shown that rapid presynaptic development can occur through the use of prefabricated protein complexes (Ahmari et al., 2000). Analogously, a mechanism for translocating modular, prefabricated core components of the postsynaptic signal transduction machinery may be required to achieve rapid development of functional spines. Conceivably, translocation of PSD-95 protein complexes may be triggered through an initial contact between filopodia and presynaptic partners (Ziv and Smith, 1996; Fiala et al., 1998), resulting in filopodial stabilization, maturation, and development into spines.

In a few examples, filopodia-spines exhibited significant structural changes after a PSD-95 cluster retracted, as if the cluster was part of a structural element that needed to be removed before the filopodium-spine could undergo this change (Fig. 4). In other cases, PSD-95 clusters were within a filopodium-spine in which the process would grow or morph around the cluster. That is, the cluster appeared to remain stable despite the surrounding process changing in shape (Fig. 2). This provides additional functional evidence that the PSD-95 cluster is a rather stable structural element.

By performing synaptophysin immunocytochemistry in combination with localization of PSD-95/GFP, we observed that, in young cultures (that have more dynamic dendritic processes), many of the clusters $(>60 \%)$ were not associated with presynaptic terminals. Perhaps these nonsynaptic clusters are reserve materials for building new synapses from prefabricated structural elements. Consistent with this idea, we found a robust increase (by approximately two times) of PSD-95/synaptophysin colocalization when analysis was restricted to PSD-95 clusters that were located in filopodia-spines. Clearly, a better understanding of the molecular events associated with the formation and maintenance of PSD-95 cluster and its role in will be crucial to understanding its role in stabilizing dendritic filopodia and spines.

\section{REFERENCES}

Ahmari SE, Buchanan J, Smith SJ (2000) Assembly of presynaptic active zones from cytoplasmic transport packets. Nat Neurosci 3:445-451.

Allison DW, Gelfand VI, Spector I, Craig AM (1998) Role of actin in anchoring postsynaptic receptors in cultured hippocampal neurons: differential attachment of NMDA versus AMPA receptors. J Neurosci 18:2423-2436.

Allison DW, Chervin AS, Gelfand VI, Craig AM (2000) Postsynaptic scaffolds of excitatory and inhibitory synapses in hippocampal neurons: maintenance of core components independent of actin filaments and microtubules. J Neurosci 20:4545-4554.

Craven SE, Bredt DS (1998) PDZ proteins organize synaptic signaling pathways. Cell 93:495-498.

Craven SE, El-Husseini AE, Bredt DS (1999) Synaptic targeting of the postsynaptic density protein PSD-95 mediated by lipid and protein motifs. Neuron 22:497-509.

Dailey ME, Smith SJ (1996) The dynamics of dendritic structure in developing hippocampal slices. J Neurosci 16:2983-2994.

Dunaevsky A, Tashiro A, Majewska A, Mason C, Yuste R (1999) Developmental regulation of spine motility in the mammalian central nervous system. Proc Natl Acad Sci USA 96:13438-13443.

El-Husseini AE, Schnell E, Chetkovich DM, Nicoll RA, Bredt DS (2000)
PSD-95 involvement in maturation of excitatory synapses. Science 290:1364-1368

Fanning AS, Anderson JM (1999) Protein modules as organizers of membrane structure. Curr Opin Cell Biol 11:432-439.

Fiala JC, Feinberg M, Popov V, Harris KM (1998) Synaptogenesis via dendritic filopodia in developing hippocampal area CA1. J Neurosci 18:8900-8911.

Fischer M, Kaech S, Knutti D, Matus A (1998) Rapid actin-based plasticity in dendritic spines. Neuron 20:847-854.

Friedman HV, Bresler T, Garner CC, Ziv NE (2000) Assembly of new individual excitatory synapses: time course and temporal order of synaptic molecule recruitment. Neuron 27:57-69.

Garner CC, Nash J, Huganir RL (2000) PDZ domains in synapse assembly and signalling. Trends Cell Biol 10:274-280.

Halpain S (2000) Actin and the agile spine: how and why do dendritic spines dance? Trends Neurosci 23:141-146.

Harris KM (1999) Structure, development, and plasticity of dendritic spines. Curr Opin Neurobiol 9:343-348.

Husi H, Ward MA, Choudhary JS, Blackstock WP, Grant SG (2000) Proteomic analysis of NMDA receptor-adhesion protein signaling complexes. Nat Neurosci 3:661-669.

Jontes JD, Smith SJ (2000) Filopodia, spines, and the generation of synaptic diversity. Neuron 27:11-14.

Lee SH, Sheng M (2000) Development of neuron-neuron synapses. Curr Opin Neurobiol 10:125-131.

Lendvai B, Stern EA, Chen B, Svoboda K (2000) Experience-dependent plasticity of dendritic spines in the developing rat barrel cortex in vivo. Nature 404:876-881.

Mackenzie PJ, Umemiya M, Murphy TH (1996) $\mathrm{Ca}^{2+}$ imaging of CNS axons in culture indicates reliable coupling between single action potentials and distal functional release sites. Neuron 16:783-795.

Okabe S, Kim HD, Miwa A, Kuriu T, Okado H (1999) Continual remodeling of postsynaptic density and its regulation by synaptic activity. Nat Neurosci 2:804-811.

Parnass Z, Tashiro A, Yuste R (2000) Analysis of spine morphological plasticity in developing hippocampal pyramidal neurons. Hippocampus 10:561-568.

Passafaro M, Sala C, Niethammer M, Sheng M (1999) Microtubule binding by CRIPT and its potential role in the synaptic clustering of PSD-95. Nat Neurosci 2:1063-1069.

Rao A, Craig AM (2000) Signaling between the actin cytoskeleton and the postsynaptic density of dendritic spines. Hippocampus 10:527-541.

Rao A, Kim E, Sheng M, Craig AM (1998) Heterogeneity in the molecular composition of excitatory postsynaptic sites during development of hippocampal neurons in culture. J Neurosci 18:1217-1229.

Saito Y, Murakami F, Song WJ, Okawa K, Shimono K, Katsumaru H (1992) Developing corticorubral axons of the cat form synapses on filopodial dendritic protrusions. Neurosci Lett 147:81-84.

Scannevin RH, Huganir RL (2000) Postsynaptic organization and regulation of excitatory synapses. Nat Rev Neurosci 1:133-141.

Segal M (2001) Rapid plasticity of dendritic spine: hints to possible functions? Prog Neurobiol 63:61-70.

Segal M, Andersen P (2000) Dendritic spines shaped by synaptic activity. Curr Opin Neurobiol 10:582-586.

Sorra KE, Harris KM (2000) Overview on the structure, composition, function, development, and plasticity of hippocampal dendritic spines. Hippocampus 10:501-511.

Topinka JR, Bredt DS (1998) N-terminal palmitoylation of PSD-95 regulates association with cell membranes and interaction with $\mathrm{K}^{+}$channel Kv1.4. Neuron 20:125-134.

Walikonis RS, Jensen ON, Mann M, Provance Jr DW, Mercer JA, Kennedy MB (2000) Identification of proteins in the postsynaptic density fraction by mass spectrometry. J Neurosci 20:4069-4080.

Wong WT, Wong RO (2000) Rapid dendritic movements during synapse formation and rearrangement. Curr Opin Neurobiol 10:118-124.

Zhang W, Benson DL (2001) Stages of synapse development defined by dependence of F-actin. J Neurosci 21:5169-5181.

Ziv NE, Smith SJ (1996) Evidence for a role of dendritic filopodia in synaptogenesis and spine formation. Neuron 17:91-102. 